\title{
3D imaging of theranostic nanoparticles in mice organs by means of $x$-ray phase contrast tomography
}

E. Longo, A. Bravin, F. Brun, I. Bukreeva, A. Cedola, et al.

E. Longo, A. Bravin, F. Brun, I. Bukreeva, A. Cedola, O. De La Rochefoucauld, M. Fratini, X. Le Guevel, L. Massimi, L. Sancey, O. Tillement, P. Zeitoun, "3D imaging of theranostic nanoparticles in mice organs by means of X-ray phase contrast tomography," Proc. SPIE 10573, Medical Imaging 2018: Physics of Medical Imaging, 105734I (9 March 2018); doi: $10.1117 / 12.2293090$

SPIE. Event: SPIE Medical Imaging, 2018, Houston, Texas, United States 


\title{
3D imaging of theranostic nanoparticles in mice organs by means of X-ray Phase Contrast Tomography
}

\author{
E. Longo ${ }^{1}$, A. Bravin ${ }^{2}$, F. Brun ${ }^{3}$, I. Bukreeva ${ }^{3}$, A. Cedola ${ }^{3}$, O. De La Rochefoucauld ${ }^{4 *}$, M. Fratini ${ }^{3,5}$, \\ X. Le Guevel ${ }^{6}$, L. Massimi ${ }^{3}$, L. Sancey ${ }^{6}$, O. Tillement ${ }^{7}$, P. Zeitoun ${ }^{1}$ \\ ${ }^{1}$ Laboratoire d'Optique Appliquée UMR7639, ENSTA-CNRS-Ecole Polytechnique-Université \\ Paris-Saclay, 181 chemin de la Hunière et des Joncherettes, 91762 Palaiseau, France \\ ${ }^{2}$ European Synchrotron Radiation Facility, 71 Avenue de Martyrs, 38043 Grenoble, France \\ ${ }^{3}$ Institute of Nanotechnology-CNR, Rome-Unit, Piazzale Aldo Moro 5 00185, Rome, Italy \\ ${ }^{4}$ Imagine Optic, Rue François Mitterrand, 33400 Talence, France \\ ${ }^{5}$ IRCCS Santa Lucia Foundation, MARBLab, Via Ardeatina 306/354, 00142, Roma, Italy \\ ${ }^{6}$ Institute for Advanced Biosciences U1209 UMR5309 UGA, Allée des Alpes - Site Santé \\ 38700 La Tronche, Grenoble, France \\ ${ }^{7}$ Institut lumière-matière, UMR5306, Université Claude Bernard Lyon1-CNRS, Université de \\ Lyon 69622 Villeurbanne, France \\ *Corresponding author: odlrochefoucauld@imagine-optic.com
}

\begin{abstract}
Theranostics is an innovative research field that aims to develop high target specificity cancer treatments by administering small metal-based nanoparticles (NPs). This new generation of compounds exhibits diagnostic and therapeutic properties due to the high atomic number of their metal component. In the framework of a combined research program on low dose X-ray imaging and theranostic NPs, X-ray Phase Contrast Tomography (XPCT) was performed at ESRF using a $3 \mu \mathrm{m}$ pixel optical system on two samples: a mouse brain bearing melanoma metastases injected with gadolinium NPs and, a mouse liver injected with gold NPs. XPCT is a non-destructive technique suitable to achieve the $3 \mathrm{D}$ reconstruction of a specimen and, widely used at micro-scale to detect abnormalities of the vessels, which are associated to the tumor growth or to the development of neurodegenerative diseases. Moreover, XPCT represents a promising and complementary tool to study the biodistribution of theranostic NPs in biological materials, thanks to the strong contrast with respect to soft tissues that metal-based NPs provide in radiological images. This work is relied on an original imaging approach based on the evaluation of the contrast differences between the images acquired below and above K-edge energies, as a proof of the certain localization of NPs. We will present different methods aiming to enhance the localization of NPs and a 3D map of their distribution in large volume of tissues.
\end{abstract}

Keywords: Nanoparticles; X-Ray Phase Contrast Tomography; 3D rendering.

\section{INTRODUCTION}

The request of tumor early diagnosis and more efficient radiotherapeutic treatments circumscribed to the only neoplastic region is demanding. Theranostics is an innovative research field merging therapeutics and diagnostics for many clinical trials, including also oncology applications [1]. Theranostics studies rely on the administration of nano-agents having an inner core with a high atomic number metal. Owing to their small size, nanoparticles (NPs) easily permeate membranes and accumulate in tumors. Thanks to their contrast properties, they allow the detection of neoplasias by means of several imaging techniques, such as Magnetic Resonance Imaging (MRI), X-ray tomography and Positron Emission Tomography (PET); and favour the destruction of tumor masses following the X-ray irradiation during radiotherapy sessions [2], [3], [4]. The design of more and more efficient theranostic NPs requires a scrupulous knowledge of their 
behaviour in tumor structures, and distribution in healthy tissues in order to exclude any toxicity effects. Various highsensitive imaging methods are in use for this purpose, such as histology, Atomic Force Microscopy (AFM), two-photon microscopy and LIBS [5], [6]. However, they share a common limitation: the irreversible disruption of the sample and lack of enough information to create high-resolution 3D models. Or, as in the case of histology and fluorescence microscopy, the use of specific staining is considered for improving the imaging properties, but at the risk of compromising the effective NPs distribution. An alternative approach that preserves the integrity of the sample and does not foresee the application of any chemical agent is X-ray tomography. Tomography allows collecting 3D information of the inner structures of an object, simply by acquiring radiographs at many angles. X-ray Phase Contrast Tomography (XPCT) is a method particularly recommended to image weak absorbing materials, such as biological samples [7]. In particular, XPCT is a well established technique to study abnormalities of the vascular system related to the onset of neurodegenerative diseases and tumors [8], [9], [10]. We propose here an innovative application of XPCT as a tool suitable to provide a 3D map of the NPs distribution in different removed mice organs. The samples, all investigated post-mortem, were a mouse brain model of multiple melanoma metastases in advanced state, in-vivo injected with gadolinium-based NPs, named AGuIX ${ }^{\circledR}$ [2], [5], and a mouse liver previously injected with ultra-small gold NPs (size smaller than $3 \mathrm{~nm}$ ) called also gold nanoclusters [11], [12], [13]. We present the preliminary results of volume reconstructions obtained with different processing methods showing a 3D map of the NPs distribution. The work is part of a project aimed to isolate the main contribution provided by NPs by evaluating the differences in the grey levels of the images above and below K-edge.

\section{METHODS}

\section{X-ray Phase Contrast Tomography (XPCT)}

Soft tissue components are characterized by poor X-ray absorption, and are difficult to be differentiated by means of standard absorption tomography. A better contrast between different material regions is essential when the different organs need to be segmented in images. XPCT is a non-invasive technique suitable to image the inner structures of biological materials, thanks to the contrast enhancement produced at the various interfaces following the phase shift induced by the imaged sample [14], [15], [16]. Up to date, various techniques have been developed in order to reach this goal, such as propagation-based imaging [17], grating interferometry [18] and edge-illumination [19]. The approach used in this work is named "in-line XPCT" and consists in leaving a propagation distance between the specimen and the detection camera according to the energy set. The method mainly relies on the X-ray refraction from the different components of an object. In the X-ray range, the refraction index is a complex number $n=1-\delta+i \beta$, where $\delta$ and $\beta$ are related to the electron density of a material. When the nearly-parallel beam passes through the sample, a distorted wavefront is captured in the radiographs and the mixed information of phase and absorption can disentangled by means of specific algorithms [7]. One of most used method for retrieving the phase information is the method proposed by Paganin [20]. It is based on the assumption that biological materials can be considered in first approximation as possessing a homogeneous composition, that quasi-monochromatic radiation is used, that paraxial approximation can be applied to transport-of-intensity equation, that the object is an optically thin sample and that the distance $\mathrm{z}$ between the rotating sample and the camera fulfils the near-field condition:

$$
z \ll d^{2} / \lambda
$$

where $d$ is the size of the smallest resolvable structure in the image and $\lambda$ the wavelength of the incident radiation. Following these assumptions, the phase map implemented in Paganin's algorithm depends on the ratio $\delta / \beta$ of the real and imaginary components of the refractive index and not on their absolute values.

\section{Mice samples}

In this work we studied ex-vivo mice brain and liver. After extraction, all the samples were stored in paraformaldehyde $4 \%$ for 4 hours at $4^{\circ} \mathrm{C}$, and stored for long time in PBS at $4^{\circ}$. 
Brain. The mouse carrying multiple brain melanoma metastases was sacrificed 1 hour after the injection of $15 \mu$ mol of $\mathrm{AGuIX}^{\circledR} \mathrm{NPs}$, a compound mainly made up of Gd and Si having diameter of $3 \mathrm{~nm}$. Sample courtesy of the experiment described in manuscript of Kotb et al [2].

Liver. The liver was explanted 5 hours after the administration of $5 \mathrm{mg} \mathrm{Au} / \mathrm{mL}(200 \mu \mathrm{L})$ of gold nanoclusters AuZwMe $\mathrm{A}_{2}$ [13] having diameter of $5 \mathrm{~nm}$.

\section{Experimental set-up}

The experiments were carried out at the biomedical beamline ID17 at the European Synchrotron Radiation Facility (ESRF) in Grenoble, France. The brain doped with AGuIX ${ }^{\circledR}$ NPs was imaged below and above gadolinium K-edge, at $48.5 \mathrm{keV}$ and $51.5 \mathrm{keV}$ respectively, whereas the tomography of the liver containing gold nanoclusters was performed close to gold K-edge, at $79.2 \mathrm{keV}$ (below K-edge) and at $82.2 \mathrm{keV}$ (above K-edge). The projections were acquired with a CMOS camera connected to an optical system determining a final pixel size of $3 \mu \mathrm{m} \times 3 \mu \mathrm{m}$. According to the quasimonochromatic incident radiation chosen, the distances sample-CMOS were fixed at $2.3 \mathrm{~m}$ for the brain and $11 \mathrm{~m}$ for the liver in order to satisfy the near-field condition. The samples were embedded in Agar-agar. Agar-agar was used to prevent the samples from moving into Eppendorf tubes. The entire volumes were scanned in half-acquisition conditions, i. e. by rotating the samples over $360^{\circ}$. The time acquisition for a single-image was $0.07 \mathrm{~s}$.

\section{Data analysis}

The acquired datasets were reconstructed by means of SYRMEP TomoProject software [21]. The tool is conceived to assist the tomographic data reconstruction from the pre-processing, including the flat-field normalization of the projection data up to the Filtered Back Projection reconstruction algorithm. It proposes also an optional phase-retrieval step essential for the reconstruction of data from single distance propagation based-experiments. Paganin's algorithm [20] was chosen to retrieve the phase map since it is a non-iterative method, stable to noise and requires to tune the only parameter $\delta / \beta$. The open-source software FIJI was used to analyse the stacks of reconstructed slices and to correct some minor artefacts, such as the oversaturation of the pixels due to the centre of rotation.

The 3D segmentation of the tissues was carried out with the open-source software VolView, which allows visualizing the different components of a material in a false scale colour by establishing a transfer function between the gray levels of the image and a colour map.

\section{RESULTS}

\section{Brain imaging}

As seen in Fig.1a and 1b, the presence of NPs enhanced the possibility of well visualizing many suspicious masses indicated by red arrows. The volume shown in Fig.1a was imaged at $51.5 \mathrm{keV}$, above gadolinium K-edge, where the contrast properties provided by gadolinium-based compounds are stronger with respect with that of a brain tissue. The volume was obtained from the sum of the maximum and minimum intensities values and it represents the reconstruction of a brain region $1.8 \mathrm{~mm}$ thick. The image shows different gray levels related to the variegated tissue composition. The dense and irregular regions displayed in white, indicated by the red arrows, are tumor masses highlighted by an abundant presence of $A G u I X^{\circledR}$ NPs. The images clearly show that the nano-agents could reach their target one hour post i.v. administration. The reconstructed images confirmed the presence of various abnormal structures, i.e. the melanoma metastases, and a high tumor visualization capability of the gadolinium-riche NPs. The image illustrates also part of the vascular system. The sample was not perfused and very presumably NPs traces also remained entrapped in the vessels, whereas no extravasation in the healthy tissue was shown. Fig. $1 \mathrm{~b}$ represents the projection of maximum intensity values of a stack of slices $765 \mu \mathrm{m}$ thick. This time the slices belong to a dataset acquired at $48.5 \mathrm{keV}$; where the contrast properties of gadolinium are still strong enough to reveal the position of tumor masses in the brain. The form according to which clusters of $\mathrm{AGuIX}^{\circledR}$ distribute in the tumor was enhanced through the projection of the maximum intensity values of a stack of slices. Fig. 1c displays the 3D reconstruction of a select region of interest of the brain carrying tumor. The colour map in false colours indicates in yellow the areas of highest density attributable to a considerable 
concentration of gadolinium in the tumor and in little amount in the vessels, according to the AGuIX® kinetics of distribution whereby the half-life time of these NPs in blood is $21.6 \mathrm{~min}$ in mice [5].
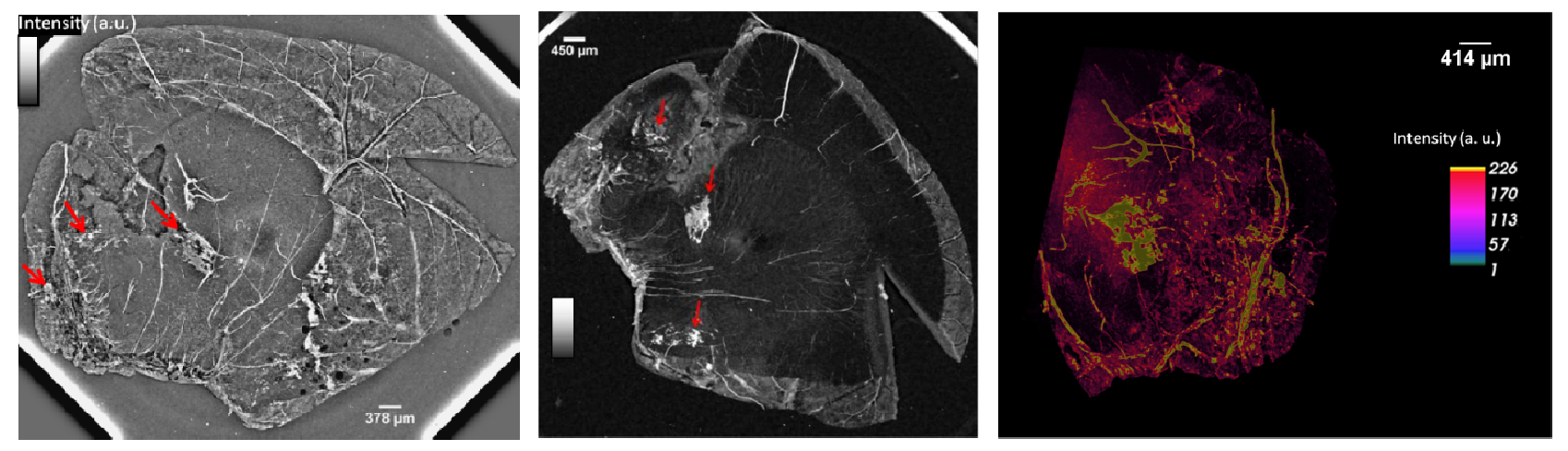

Figure 1. (a) On the left, volume reconstruction of $1.8 \mathrm{~mm}$ thick of mouse brain. Red arrows indicate the multiple melanoma metastases originated in this region of the brain. Their very dense appearance indicated with white colour is due to a high concentration of $\mathrm{AGuIX}^{\mathbb{R}}$ NPs. (b) In the centre, maximum intensity projection image of the brain using a $765 \mu \mathrm{m}$ thick total sample thickness. (c) On the right, 3D segmented visualization of a small region of interest of the brain with tumor. As indicated from the colour map, green/yellow colours correspond to the densest regions: gadolinium NPs targeted the tumor and discrete amount of nanoagents remained entrapped in the vascular network.

\section{Liver imaging}

A section of the vascular network of the liver is represented in Fig. 2. It was obtained using the software FIJI by combining the maximum and minimum intensity projections of a selected number of slices. The image shows two main veins, recognizable for their large diameters and wide bifurcations, surrounded by a tangled net of peripheral arteries. Fig.3 shows four different views of the 3D segmented rendering of the entire mouse liver. The 3D reconstruction was obtained with the software VolView from XPCT dataset. As it is displayed, a non-uniform yellow patina coats the liver surface and very dense regions were detected also in the liver tissue and visualized in orange. These could be the signatures of the scattered homogeneous signal produced by gold NPs retained by the liver 5 hours after injection. Data treatment is still under progress to confirm the gold NPs localization.

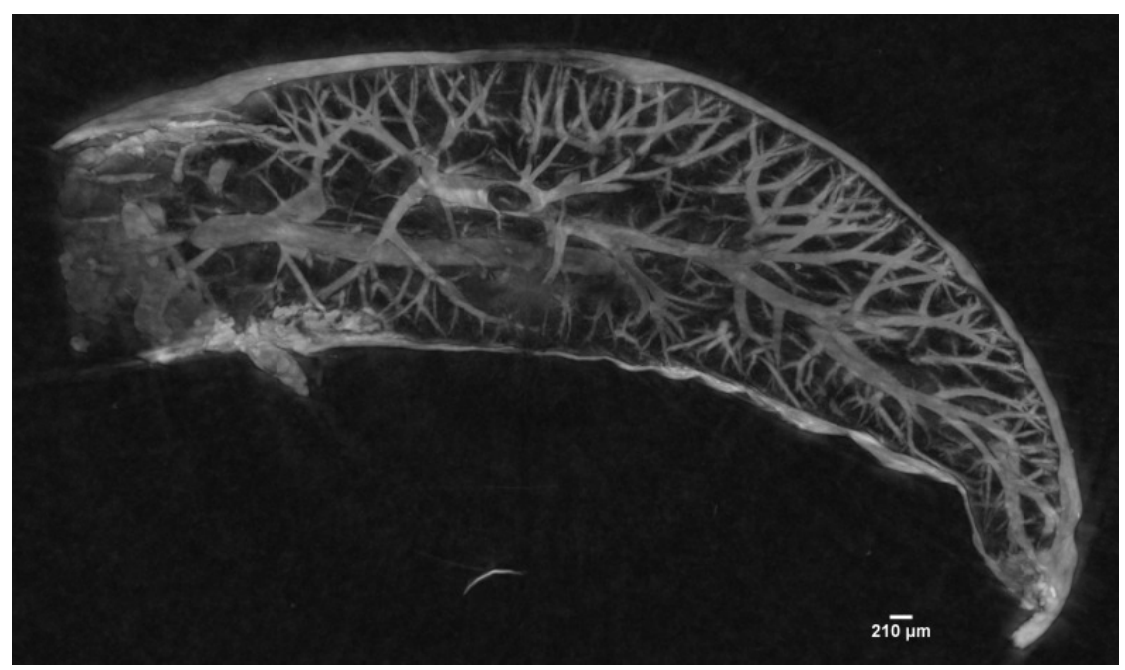

Figure 2. 3D segmentation of the mouse hepatic vascular system. 

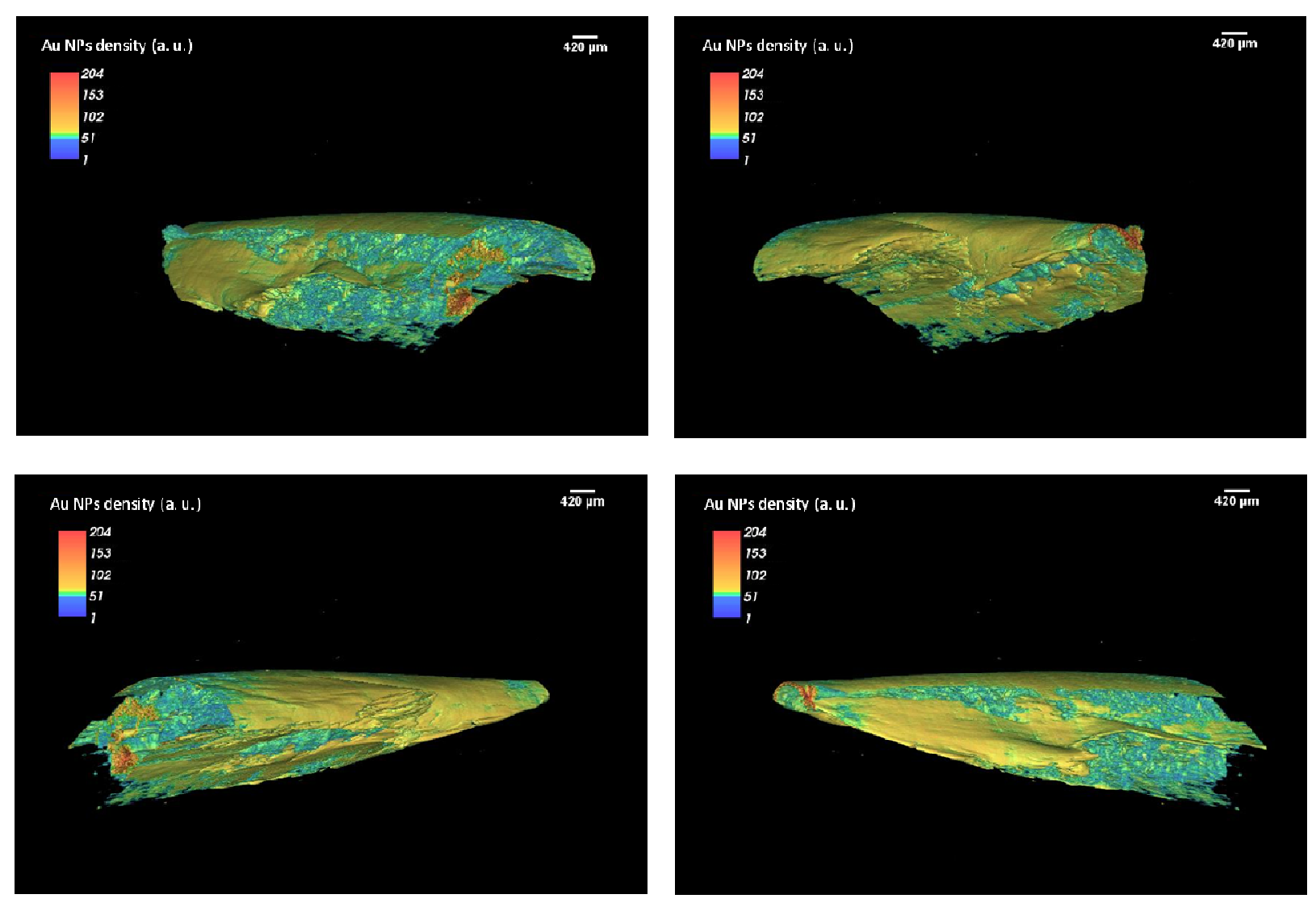

Figure 3. 3D false colour rendering of a mouse liver showing in yellow colour gold nanoclusters deposited on the external surface of the liver and in orange the strong concentration retained in the inner tissues.

\section{CONCLUSIONS}

XPCT has proven to be an adaptable technique for different kind of investigations. High resolution XPCT allowed accessing simultaneously to i) the vascular system, even up to small structures in the range of few micrometers; ii) locate the accumulations of NPs in healthy and cancer tissues; and iii) achieve 3D visualization of an object. XPCT

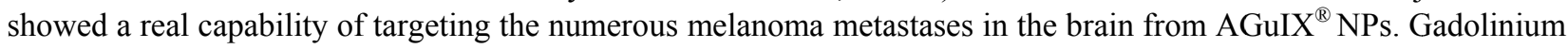
showed strong contrast properties both below and above K-edge, by providing an enhancement of the tumor structures at both chosen energies. The results obtained showed that XPCT can be considered as a complementary approach next to other well-established and sensitive techniques used for the imaging of theranostic NPs in biologic tissues. By combining different methods in the post-processing, such as the projection of maximum and minimum intensities and segmenting the image helped in getting information about the location of NPS in brain and liver tissues. The reconstructed images seem encouraging to deepen the studies of distribution of NPs by means of XPCT.

\section{ACKNOWLEDGEMENTS}

This work was partially funded by the VOXEL project (European Union's Horizon 2020 research and innovation program under grant agreement $\mathrm{N}^{\circ}$ 665207") and COST Action MP1203. 


\section{REFERENCES}

[1] A. Mignot et al., "A top-down synthesis route to ultrasmall multifunctional Gd-based silica nanoparticles for theranostic applications," Chem. - A Eur. J., vol. 19, no. 19, pp. 6122-6136, 2013.

[2] S. Kotb et al., "Gadolinium-Based Nanoparticles and Radiation Therapy for Multiple Brain Melanoma Metastases: Proof of Concept before Phase I Trial.," Theranostics, vol. 6, no. 3, pp. 418-427, 2016.

[3] S. Dufort et al., "Nebulized gadolinium-based nanoparticles: A theranostic approach for lung tumor imaging and radiosensitization," Small, vol. 11, no. 2, pp. 215-221, 2015.

[4] L. Sancey et al., "The use of theranostic gadolinium-based nanoprobes to improve radiotherapy efficacy," $B r . J$. Radiol., vol. 87, no. 1041, pp. 1-15, 2014.

[5] L. Sancey et al., "Long-term in Vivo clearance of gadolinium-based AGuIX nanoparticles and their biocompatibility after systemic injection," ACS Nano, vol. 9, no. 3, pp. 2477-2488, 2015.

[6] L. Sancey et al., "Laser spectrometry for multi-elemental imaging of biological tissues," Sci. Rep., vol. 4, p. 6065, 2014.

[7] A. Bravin, P. Coan, and P. Suortti, "X-ray phase-contrast imaging: From pre-clinical applications towards clinics," Phys. Med. Biol., vol. 58, no. 1, 2013.

[8] A. Cedola et al., "X-Ray Phase Contrast Tomography Reveals Early Vascular Alterations and Neuronal Loss in a Multiple Sclerosis Model," Sci. Rep., vol. 7, no. 1, pp. 1-11, 2017.

[9] M. Fratini et al., "Simultaneous submicrometric 3D imaging of the micro-vascular network and the neuronal system in a mouse spinal cord.,"Sci. Rep., vol. 5, p. 8514, 2015.

[10] F. Pfeiffer et al., "High-resolution brain tumor visualization using three-dimensional x-ray phase contrast tomography," Phys. Med. Biol., vol. 52, no. 23, pp. 6923-6930, 2007.

[11] R. Jin, C. Zeng, M. Zhou, and Y. Chen, "Atomically Precise Colloidal Metal Nanoclusters and Nanoparticles: Fundamentals and Opportunities," Chem. Rev., vol. 116, no. 18, pp. 10346-10413, 2016.

[12] X. Le Guevel, "Recent advances on the synthesis of metal quantum nanoclusters and their application for bioimaging," IEEE J. Sel. Top. Quantum Electron., vol. 20, no. 3, 2014.

[13] D. Shen et al., "Zwitterion functionalized gold nanoclusters for multimodal near infrared fluorescence and photoacoustic imaging," APL Mater., vol. 5, no. 5, 2017.

[14] S. W. Wilkins, "Phase-contrast imaging using polychromatic hard x-rays," Nature, vol. 384, pp. 335-8, 1996.

[15] R. Fitzgerald, “P HASE -S ENSITIVE X-R AY I MAGING,” pp. 23-26, 2000.

[16] A. Snigirev, I. Snigireva, V. Kohn, S. Kuznetsov, and I. Schelokov, "On the possibilities of x-ray phase contrast by coherent high-energy synchrotron radiation,” Rev. Sci. Instrum., vol. 66, no. 12, pp. 5486-5492, 1995.

[17] T. Weitkamp, D. Haas, D. Wegrzynek, and A. Rack, "ANKAphase: Software for single-distance phase retrieval from inline X-ray phase-contrast radiographs," J. Synchrotron Radiat., vol. 18, no. 4, pp. 617-629, 2011.

[18] T. Weitkamp et al., "X-ray phase imaging with a grating interferometer," Opt. Express, vol. 13, no. 16, p. 6296, 2005.

[19] P. C. Diemoz, C. K. Hagen, M. Endrizzi, and A. Olivo, "Sensitivity of laboratory based implementations of edge illumination X-ray phase-contrast imaging," Appl. Phys. Lett., vol. 103, no. 24, 2013. 
[20] D. Paganin, S. C. Mayo, T. E. Gureyev, P. R. Miller, and S. W. Wilkins, "Simultaneous phase and amplitude extraction from a single defocused image of a homogeneous object," J. Microsc., vol. 206, no. 1, pp. 33-40, 2002.

[21] F. Brun et al., "SYRMEP Tomo Project: a graphical user interface for customizing CT reconstruction workflows," Adv. Struct. Chem. Imaging, 2017. 